Rev. Int. Contam. Ambie. 37, 307-318, 2021

https://doi.org/10.20937/RICA.53879

\title{
OPTIMIZACIÓN DE LAS VARIABLES DEL PROCESO DE DIGESTIÓN ANAEROBIA DE LACTOSUERO EN LA PRODUCCIÓN DE BIOGÁS
}

Optimization of variables from the anaerobic digestion process of cheese whey in biogas production

Sergio CISNEROS DE LA CUEVA ${ }^{1 *}$, Fabiola VEANA HERNÁNDEZ ${ }^{2}$, Marco Antonio ARJONA LÓPEZ ${ }^{3}$, Cecilia Lizeth ALVAREZ GUZMÁN ${ }^{4}$ y Samuel Bernardo PÉREZ VEGA ${ }^{1}$

\footnotetext{
${ }^{1}$ Facultad de Ciencias Químicas, Universidad Autónoma de Chihuahua, Campus UACH II, Circuito Universitario s/n, 31125 Chihuahua, Chihuahua, México.

2 Tecnológico Nacional de México/IT de Ciudad Valles, km 2 Carretera al Ingenio Plan de Ayala, Vista Hermosa, 79010 Ciudad Valles, San Luis Potosí, México.

${ }^{3}$ Tecnológico Nacional de México/IT de La Laguna, Blvd. Revolución y Av. I.T. Laguna s/n, 27000 Torreón, Coahuila, México.

${ }^{4}$ Instituto Potosino de Investigación Científica y Tecnológica A.C., Camino a la Presa de San José 2055, Lomas 4a Sección, 78216 San Luis Potosí, San Luis Potosí, México.
}

*Autor para correspondencia: ser081083@gmail.com

(Recibido: febrero de 2020; aceptado: julio de 2020)

Palabras clave: bioprocesos, combustibles fósiles, diseño de optimización experimental, residuos agroindustriales

\section{RESUMEN}

Con el objetivo de reducir el consumo de combustibles fósiles y tener biogás con precios competitivos en el mercado energético, es necesario disponer de bioprocesos económicos y de altos rendimientos de producción. En este estudio, se demuestra que la producción de biogás y el rendimiento de metano pueden incrementarse optimizando algunas de las variables del proceso de digestión anaeróbica del lactosuero. Las condiciones iniciales de las variables de proceso, como concentración de sustrato, concentración de inóculo y $\mathrm{pH}$, fueron evaluadas y optimizadas mediante un diseño central compuesto. Las condiciones óptimas para los máximos valores de rendimiento de metano $\left(469.75 \pm 3.25 \mathrm{~mL} \mathrm{CH}_{4} / \mathrm{g} \mathrm{SV}\right)$ y producción de biogás (331 $\pm 1.13 \mathrm{~mL}$ ) fueron: concentración inicial de sustrato de $24.6 \mathrm{~g} / \mathrm{L}$, concentración inicial de inóculo de $22.4 \mathrm{~g} / \mathrm{L}$ y pH inicial de 7.1. El análisis de los ácidos grasos volátiles indicó que la formación de metano en el biogás se debe principalmente a la transformación metabólica del acetato por la vía de la metanogénesis ecetoclástica. Los resultados obtenidos muestran que al utilizar lactosuero como sustrato y en las condiciones óptimas obtenidas, el bioproceso desarrollado en este estudio puede ser una nueva opción biotecnológica industrial para la producción de biogás.

Key words: agro-industrial wastes, bioprocesses, experimental optimization design, fossil fuels

\begin{abstract}
To reduce the consumption of fossil fuels and to have competitive prices of biogas in the energy market, it is necessary to have bioprocesses with low cost and high efficiency of
\end{abstract}


production. This study demonstrates that biogas production and methane yield can be increased optimizing some of the variables of the anaerobic digestion process of cheese whey. The initial conditions of process variables, such as initial substrate concentration, initial inoculum concentration, and initial $\mathrm{pH}$ were evaluated and optimized using a central composite design. The optimal conditions for the maximum values of methane yield $\left(469.75 \pm 3.25 \mathrm{~mL} \mathrm{CH}_{4} / \mathrm{g} \mathrm{SV}\right)$ and biogas production $(331 \pm 1.13 \mathrm{~mL})$ were the following: initial substrate concentration of $24.6 \mathrm{~g} / \mathrm{L}$, initial inoculum concentration of $22.4 \mathrm{~g} / \mathrm{L}$, and initial $\mathrm{pH}$ of 7.1 . The analysis of volatile fatty acids indicated that the formation of methane in biogas is mainly due to metabolic transformation of acetate by the acetoclastic methanogenesis pathway. The obtained results show that using cheese whey as substrate and under the optimal conditions obtained, the bioprocess developed in this study can be a new industrial biotechnological option for the production of biogas.

\section{INTRODUCCIÓN}

La emisión antrópica de gases de efecto invernadero generada por el uso de combustibles fósiles es considerada la principal causa de contaminación ambiental (Carrillo et al. 2014, Wang et al. 2017). El gas natural (GN) es un combustible fósil finito que, debido a su alto consumo, se está agotando y sus costos se están elevando (Burke y Yang 2016, IEA 2019). En 2018, el consumo de GN a nivel mundial fue de 3848.9 billones de metros cúbicos (Dudley 2019). Algunos escenarios energéticos mundiales asumen que para 2050 el abastecimiento de GN se hará a partir de fuentes de energía renovables (Council 2019).

El biogás es una fuente importante de energía renovable que ayuda a mitigar la emisión de gases de efecto invernadero y a disminuir el consumo de combustibles fósiles (Saleh et al. 2012). Se obtiene de la digestión anaerobia llevada a cabo por microorganismos (Deublein y Steinhauser 2008), la cual es un proceso de degradación biológica de compuestos orgánicos (carbohidratos, proteínas y lípidos) que se lleva a cabo en cuatro etapas: hidrólisis, acidogénesis, acetogénesis y metanogénesis (Kim et al. 2010, Liu et al. 2012). Aunque la composición del biogás varía, el metano $\left(\mathrm{CH}_{4}\right)$ es el compuesto de mayor proporción con un rango de 55-70\%, seguido por el dióxido de carbono $\left(\mathrm{CO}_{2}\right)$ con un rango de 30-40 \% y otros gases en cantidades traza (Deublein y Steinhauser 2008, Saleh et al. 2012). El biogás se puede usar como energía de calentamiento y para la generación de electricidad y propulsión de máquinas térmicas, mientras que los subproductos (líquidos y sólidos) se pueden usar como fertilizantes en la agricultura (Leung y Wang 2016).

El lactosuero es uno de los principales residuos de la industria láctea, particularmente en la producción de queso, ya que para obtener $1 \mathrm{~kg}$ de producto se necesitan $10 \mathrm{~kg}$ de leche, de los cuales $9 \mathrm{~kg}$ terminan como lactosuero. La producción de lactosuero durante los últimos 21 años ha aumentado cerca del $3 \%$ anual. En el 2016 se produjeron aproximadamente 200 millones de toneladas a nivel mundial (Lappa et al. 2019). Debido a su alto contenido de materia orgánica y baja alcalinidad, la eliminación del lactosuero en cuerpos de agua y suelos representa una importante fuente de contaminación ambiental (Najafpour et al. 2010, Rico et al. 2015). El lactosuero se compone de lactosa (45-50 g/L), proteínas solubles $(6-8 \mathrm{~g} / \mathrm{L})$, lípidos $(4-5 \mathrm{~g} / \mathrm{L})$ y sales minerales $(8-10 \%$ extracto seco), características que lo hacen un buen sustrato para la producción de biogás por digestión anaerobia (Chatzipaschali y Stamatis 2012). Se han reportado numerosos estudios sobre el proceso de digestión anaerobia en la producción de biogás utilizando diferentes tipos de biomasa, como los monosustratos; sin embargo, el uso directo de sustratos es difícil debido a su desequilibrio nutricional, falta de microorganismos y al efecto de los factores operacionales del proceso de digestión anaerobia (Hagos et al. 2017).

Para mejorar la eficiencia de la digestión anaerobia a partir del sustrato agroindustrial y aumentar la producción de biogás, se deben optimizar variables como la relación carbono/nitrógeno, temperatura, $\mathrm{pH}$, ácidos grasos volátiles, pretratamientos de la materia prima, mezclado, concentración de inóculo y tipo de reactor (Reungsang et al. 2012, Boboescu et al. 2014). Hasta la fecha existe poca información relacionada con el análisis de optimización y control de las variables operativas del proceso de digestión anaerobia del lactosuero en la producción de biogás. En el cuadro I se muestra un resumen de la revisión de la literatura realizada.

Los métodos de optimización son técnicas matemáticas que permiten encontrar los valores óptimos de un conjunto de variables que maximizan o 
CUADRO I. VALORES DE RENDIMIENTO DE METANO REPORTADOS PARA ESTUDIOS DE DIGESTIÓN ANAEROBIA QUE USARON LACTOSUERO COMO SUSTRATO EN DIFERENTES CONDICIONES DE OPERACIÓN.

\begin{tabular}{|c|c|c|c|c|c|c|c|}
\hline $\begin{array}{l}\text { Tipo de } \\
\text { reactor }\end{array}$ & Inóculo & $\begin{array}{l}\text { Alimentación en } \\
\text { reactor } \\
\text { (S/I, VCO) }\end{array}$ & $\mathrm{pH}$ & $\begin{array}{c}\mathrm{T} \\
\left({ }^{\circ} \mathrm{C}\right)\end{array}$ & $\begin{array}{l}\text { TRH } \\
\text { (días) }\end{array}$ & $\begin{array}{l}\text { Rendimiento de } \\
\text { metano } \\
\left(\mathrm{mL} \mathrm{CH}_{4} / \mathrm{g}\right)\end{array}$ & Referencia \\
\hline RASL* & ARP & $\begin{array}{c}80 / 20 \\
(\% \text { VTR) }\end{array}$ & 7.4 & 32 & - & $\begin{array}{l}270 \\
(\mathrm{SV})\end{array}$ & Antonelli et al. (2016) \\
\hline $\mathrm{DA}^{*}$ & EAC & $\begin{array}{l}5.9 / 5.5 \\
(\% \mathrm{ST})\end{array}$ & $6.5-7.5$ & 37 & - & $\begin{array}{l}223 \\
(\mathrm{SV})\end{array}$ & Carlini et al. (2015) \\
\hline $\mathrm{DA}^{* * *}$ & LTAR & - & 7.4-7.9 & 35 & 14 & $\begin{array}{l}211 \\
\text { (SV) }\end{array}$ & Comino et al. (2009) \\
\hline $\mathrm{DA}^{* * * *}$ & EV & - & $6-7.4$ & 35 & - & $\begin{array}{l}340 \\
(\mathrm{SV})\end{array}$ & Comino et al. (2012) \\
\hline RAFA** & LTAR & $\begin{array}{c}24.6 \\
\text { (g DQO/mL día) }\end{array}$ & $7-8$ & 35 & 2.4 & $\begin{array}{c}424 \\
(\mathrm{DQO})\end{array}$ & Ergüder et al. (2001) \\
\hline RASL* & LTAR & $\begin{array}{c}1.5 \\
\text { (g DQO/mL día) }\end{array}$ & 7 & 35 & 8.3 & $\begin{array}{c}314 \\
\text { (DQO) }\end{array}$ & Fernández et al (2015) \\
\hline $\mathrm{DA}^{*}$ & EP & - & $8.7-8.5$ & 36.5 & - & $\begin{array}{l}321 \\
\text { (SV) }\end{array}$ & Kim y Kim (2017) \\
\hline $\mathrm{DA}^{*}$ & LB & $\begin{array}{c}0.06 / 0.07 \\
(\% \mathrm{SV})\end{array}$ & $5.5-5.8$ & 35 & - & $\begin{array}{l}22.8-36.3 \\
\text { (DQO) }\end{array}$ & Mainardis et al. (2017) \\
\hline $\mathrm{RCTA} * * *$ & LB & $\begin{array}{c}1.9-2.5 \\
\text { (g SV/mL día) }\end{array}$ & $7.8-8.1$ & 37 & $45-60$ & $\begin{array}{l}381.2 \\
(\mathrm{SV})\end{array}$ & Ramos et al. (2019) \\
\hline RAFA* & LTAR & $\begin{array}{c}31 \\
\text { (g DQO/mL día) }\end{array}$ & 6.7 & 35 & 0.07 & $\begin{array}{c}320 \\
(\mathrm{DQO})\end{array}$ & Rico et al. (1991) \\
\hline RHA* & LTAR & $\begin{array}{c}6.1 \\
\text { (g DQO/mL día) }\end{array}$ & $6.8-7.8$ & 35 & 1.7 & $\begin{array}{c}350 \\
(\mathrm{DQO})\end{array}$ & Strydom et al. (2001) \\
\hline
\end{tabular}

ARP: aguas residuales porcinas, DA: digestor anaerobio, DQO: demanda química de oxígeno, EAC: estiércol de aves de corral, EP: estiércol porcino, EV: estiércol de vaca, LB: lodos de biodigestor, LTAR: lodos de tratamiento de aguas residuales, RAFA: reactor anaerobio de flujo ascendente, RASL: reactor anaerobio secuencial por lotes, RCTA: reactor continuo de tanque agitado, RHA: reactor híbrido anaerobio, ST: sólidos totales, SV: sólidos volátiles, S/I: sustrato/inóculo, T: temperatura, TRH: tiempo de retención hidráulico, VCO: velocidad de carga orgánica, VTR: volumen total del reactor.

*Una fase de tratamiento anaerobio, $* *$ dos fases de tratamiento anaerobio, $* * *$ tres fases de tratamiento anaerobio, $* * * *$ cuatro fases de tratamiento anaerobio.

minimizan alguna función de interés. El objetivo de estos métodos es mejorar el rendimiento de un sistema, proceso o producto para obtener el máximo beneficio (Bezerra et al. 2008). La metodología de superficie de respuesta es una técnica estadística frecuentemente utilizada en los procesos de digestión anaerobia en el modelado y determinación de las condiciones óptimas de operación (Chun et al. 2015, Sathish y Vivekanandan 2016). Antes de emplear la metodología de superficie de respuesta es necesario elegir un diseño experimental que defina cuáles experimentos deben llevarse a cabo. Entre los diseños experimentales más utilizados para evaluar y optimizar las diferentes condiciones operativas del proceso de digestión anaerobia para la producción de biogás figuran el diseño central compuesto, el Plackette-Burman y el Box-Behnken (Wang y Wan 2009, Saleh et al. 2012, Boboescu et al. 2014).

Desde esta perspectiva, el objetivo del presente estudio fue optimizar la concentración inicial de sustrato, la concentración inicial de inóculo y el $\mathrm{pH}$ inicial en el proceso de digestión anaerobia del lactosuero mediante un análisis de superficie de respuesta. 


\section{MATERIALES Y MÉTODOS}

\section{Características del sustrato y el inóculo}

El sustrato utilizado fue lactosuero en polvo obtenido de una planta procesadora de lácteos ubicada en Torreón, Coahuila, México. El inóculo utilizado fue estiércol líquido de vaca, tomado directamente del tanque de almacenamiento de estiércol que alimenta un biodigestor de $1500 \mathrm{~m}^{3}$ de capacidad en una planta de digestión anaerobia ubicada en Gómez Palacio, Durango, México. El estiércol líquido se filtró usando un tamiz de $3 \mathrm{~mm}$ de tamaño de poro para eliminar los materiales sólidos (Antonelli et al. 2016). En el cuadro II se muestra la composición física y química del sustrato y el inóculo.

CUADRO II. COMPOSICIÓN FÍSICA Y QUÍMICA DEL SUSTRATO Y EL INÓCULO

\begin{tabular}{lrc}
\hline Parámetros & $\begin{array}{c}\text { Sustrato } \\
\text { (lactosuero) }\end{array}$ & $\begin{array}{c}\text { Inóculo } \\
\text { (estiércol líquido } \\
\text { de vaca) }\end{array}$ \\
\hline Carbohidratos totales $(\mathrm{g} / \mathrm{L})$ & $59.90 \pm 0.10$ & $4.53 \pm 0.40$ \\
Fósforo total $(\mathrm{g} / \mathrm{L})$ & $0.24 \pm 0.02$ & $0.36 \pm 0.16$ \\
Nitrógeno total $(\mathrm{g} / \mathrm{L})$ & $0.71 \pm 0.01$ & $1.76 \pm 0.12$ \\
pH & $6.52 \pm 0.09$ & $7.12 \pm 0.00$ \\
Proteínas $(\mathrm{g} / \mathrm{L})$ & $4.43 \pm 0.50$ & $14.77 \pm 0.57$ \\
Sólidos volátiles $(\mathrm{g} / \mathrm{L})$ & $59.73 \pm 0.50$ & $11.42 \pm 0.19$ \\
Sólidos totales $(\mathrm{g} / \mathrm{L})$ & $81.965 \pm 0.57$ & $21.95 \pm 0.83$ \\
\hline
\end{tabular}

\section{Diseño experimental}

Se utilizó un diseño experimental central compuesto para encontrar las condiciones óptimas de las variables, la concentración inicial de sustrato, la concentración inicial de inóculo y el pH inicial que maximizaran la producción de biogás y el rendimiento de metano. Los valores de los niveles -1 (bajo) y +1 (alto) de cada variable para estructurar el diseño experimental fueron: concentración inicial de sustrato, 12$35 \mathrm{~g} / \mathrm{L}$; concentración inicial de inóculo, 10-30 g/L, y $\mathrm{pH}$ inicial, 5.2-9.8. Las variables de respuesta fueron: producción de biogás expresado en $\mathrm{mL}$ y rendimiento de metano expresado en $\mathrm{mL} \mathrm{CH}_{4} / \mathrm{g} \mathrm{SV}$. Se realizó un total de 24 tratamientos (Cuadro III) en botellas de vidrio de $150 \mathrm{~mL}$ usando $100 \mathrm{~mL}$ para llevar a cabo el proceso de digestión anaerobia. Se utilizó un medio mineral que contiene nutrientes de crecimiento y $8 \mathrm{~g} / \mathrm{L}$ de $\mathrm{CaCO}_{3}$. Las concentraciones de los nutrientes (expresadas en $\mathrm{g} / \mathrm{L}$ ) fueron descritas por Gómez et al. 2012: $\mathrm{NaH}_{2} \mathrm{PO}_{4} \bullet \mathrm{H}_{2} \mathrm{O}, 0.703$; $\mathrm{K}_{2} \mathrm{HPO}_{4}, 0.600 ; \mathrm{NH}_{4} \mathrm{Cl}, 0.280 ; \mathrm{MgSO}_{4} \cdot 7 \mathrm{H}_{2} \mathrm{O}, 0.111$; $\mathrm{CaCl}_{2}, 0.006$, y extracto de levadura, 0.02 .
Para ajustar el pH inicial se utilizaron $\mathrm{NaOH} 5 \mathrm{~N}$ o $\mathrm{HCl} 3 \mathrm{~N}$ de acuerdo con los tratamientos descritos en el cuadro III. Para inducir las condiciones anaerobias, el espacio de cabeza de las botellas fue purgado con una mezcla de $25 \%$ de $\mathrm{CO}_{2}$ y $75 \%$ de gas $\mathrm{N}_{2}$ durante $4 \mathrm{~min}$. Las botellas se agitaron a 150 revoluciones por minuto a $35^{\circ} \mathrm{C}$ durante 17 días en una incubadora Shel Lab.

\section{Métodos analíticos}

Se usaron los métodos estándar de la APHA para determinar el $\mathrm{pH}$ (APHA 4500- $\mathrm{H}^{+}$), sólidos totales (ST) (APHA 2540-B), sólidos volátiles (SV) (APHA 2540-E), nitrógeno total (APHA 4500-Norg-C), proteínas (APHA 4500-Norg-C), y fósforo total (APHA 4500-P-B y APHA 4500-P-C) (APHA 2017). Los carbohidratos totales se determinaron por el método colorimétrico de fenol-ácido sulfúrico. Se utilizaron $2 \mathrm{~mL}$ de muestra de los tratamientos, que fueron mezclados con $1 \mathrm{~mL}$ de solución de fenol al $5 \%$ en un tubo de ensayo. Posteriormente se añadieron $5 \mathrm{~mL}$ de ácido sulfúrico concentrado a la mezcla. Después los tubos de ensayo se dejaron en reposo durante $10 \mathrm{~min}$. Pasado el tiempo, los tubos se agitaron vorticialmente durante $30 \mathrm{~s}$ y se colocaron durante 20 min en un baño de agua a temperatura ambiente. Luego, a los tubos se les midió la absorbancia en un espectrofotómetro UVVIS 6405, Jenway a una longitud de onda de $490 \mathrm{~nm}$ (Albalasmeh et al. 2013). Para la cuantificación de los AGV (acetato, propionato y butirato), se filtraron $2 \mathrm{~mL}$ de muestra de los diferentes tratamientos usando un filtro Whatman de $0.45 \mu \mathrm{m}$ para ser analizados en un cromatógrafo de líquidos (HPLC) equipado con columna (Agilent Hi-Plex H, $7.7 \times$ $300 \mathrm{~mm}, 8 \mu \mathrm{m}$ [p/n PL1170-6830]) y detector de índice de refracción (RID). Se utilizó una solución de $0.1 \mathrm{M}$ de $\mathrm{H}_{2} \mathrm{SO}_{4}$ como fase móvil con un flujo de $0.6 \mathrm{~mL} / \mathrm{min}$. Durante el análisis las temperaturas de la columna y RID se mantuvieron a 60 y $55^{\circ} \mathrm{C}$, respectivamente.

Para la determinación del volumen de biogás y metano, a cada botella se le conectó una manguera de silicón Masterflex de $2 \mathrm{~mm}$ de diámetro, la cual permitió la salida del gas alojado en el espacio vacío de la botella. El gas desalojado se hizo pasar por un medidor de flujo de gas MilliGascounters Ritter para cuantificar el volumen de biogás; seguidamente se dejó pasar el gas hasta una bureta invertida que contenía una solución de $\mathrm{NaOH} 1 \mathrm{~N}$. El alojamiento del gas en la bureta provocó el desplazamiento del $\mathrm{NaOH} 1 \mathrm{~N}$ y de esta manera se cuantificó el volumen de metano, ya que el $\mathrm{CO}_{2}$ y otros gases quedan atrapados en la solución de $\mathrm{NaOH} 1 \mathrm{~N}$ (Rosales et al. 2010, Scherer 
CUADRO III. PRODUCCIÓN DE BIOGÁS Y RENDIMIENTO DE METANO DE LOS TRATAMIENTOS CON DIFERENTES NIVELES EN LAS VARIABLES INDEPENDIENTES A PARTIR DEL DISEÑO EXPERIMENTAL CENTRAL COMPUESTO.

\begin{tabular}{|c|c|c|c|c|c|}
\hline \multirow[b]{2}{*}{ Tratamiento } & \multicolumn{3}{|c|}{ Condiciones iniciales } & \multicolumn{2}{|c|}{ Variables de respuesta } \\
\hline & Inóculo $(\mathrm{g} / \mathrm{L})$ & $\begin{array}{l}\text { Sustrato } \\
(\mathrm{g} / \mathrm{L})\end{array}$ & $\mathrm{pH}$ & $\begin{array}{l}\text { Biogás } \\
(\mathrm{mL})\end{array}$ & $\begin{array}{c}\text { Metano } \\
\left(\mathrm{mL} \mathrm{CH}_{4} / \mathrm{g} \mathrm{SV}\right)\end{array}$ \\
\hline 1 & 30 & 35 & 9.8 & $122 \pm 5.65$ & $95.73 \pm 1.25$ \\
\hline 2 & 20 & 23.5 & 7.5 & $331 \pm 2.82$ & $471.50 \pm 2.77$ \\
\hline 3 & 30 & 35 & 5.2 & $179 \pm 9.89$ & $124.22 \pm 6.45$ \\
\hline 4 & 40 & 23.5 & 7.5 & $304 \pm 4.24$ & $292.01 \pm 3.04$ \\
\hline 5 & 20 & 23.5 & 7.5 & $330 \pm 1.41$ & $472.10 \pm 0.19$ \\
\hline 6 & 30 & 12 & 5.2 & $176 \pm 6.36$ & $172.24 \pm 4.19$ \\
\hline 7 & 20 & 23.5 & 12.1 & $0 \pm 0$ & $0 \quad \pm 0$ \\
\hline 8 & 30 & 35 & 5.2 & $193 \pm 0.70$ & $139.11 \pm 0.84$ \\
\hline 9 & 20 & 23.5 & 2.9 & $0 \pm 0$ & $0 \quad \pm 0$ \\
\hline 10 & 30 & 12 & 9.8 & $84 \pm 4.94$ & $35.21 \pm 2.31$ \\
\hline 11 & 30 & 12 & 5.2 & $194 \pm 4.10$ & $201.54 \pm 2.32$ \\
\hline 12 & 20 & 46.5 & 7.5 & $276 \pm 2.68$ & $134.06 \pm 1.15$ \\
\hline 13 & 10 & 35 & 9.8 & $102 \pm 3.67$ & $86.75 \pm 2.82$ \\
\hline 14 & 10 & 35 & 5.2 & $197 \pm 3.53$ & $149.56 \pm 3.33$ \\
\hline 15 & 10 & 12 & 9.8 & $102 \pm 0.42$ & $104.72 \pm 0.66$ \\
\hline 16 & 10 & 12 & 5.2 & $163 \pm 2.26$ & $163.24 \pm 2.74$ \\
\hline 17 & 10 & 12 & 9.8 & $103 \pm 1.13$ & $110.48 \pm 0$ \\
\hline 18 & 10 & 12 & 5.2 & $151 \pm 0.56$ & $155.23 \pm 0.13$ \\
\hline 19 & 30 & 12 & 9.8 & $87 \pm 0.56$ & $93.63 \pm 0.69$ \\
\hline 20 & 30 & 35 & 9.8 & $88 \pm 2.12$ & $74.26 \pm 2.45$ \\
\hline 21 & 10 & 35 & 9.8 & $75 \pm 0.28$ & $66.39 \pm 1.52$ \\
\hline 22 & 0 & 23.5 & 7.5 & $0 \pm 0$ & $0 \quad \pm 0$ \\
\hline 23 & 10 & 35 & 5.2 & $174 \pm 3.25$ & $129.22 \pm 1.24$ \\
\hline 24 & 20 & 0.5 & 7.5 & $66 \pm 4.38$ & $35.80 \pm 0.92$ \\
\hline
\end{tabular}

y Neumann 2013). Para determinar de concentración de metano y $\mathrm{CO}_{2}$ se utilizaron los sensores MQ-4 y MG811, respectivamente; ambos se colocaron en la entrada de flujo de gas de la bureta invertida y se conectaron a una tarjeta de adquisición de datos conectada a una computadora, la cual mostraba y registraba los datos en tiempo real.

\section{Análisis estadístico}

El análisis de regresión de los datos experimentales obtenidos a partir del diseño central compuesto se llevó a cabo con un modelo heurístico polinomial de segundo orden (ecuación 1), con la intención de obtener las condiciones óptimas.

$$
\begin{aligned}
Y= & \beta_{0}+\sum_{\mathrm{i}=1}^{\mathrm{k}} \beta_{i} X_{i}+\sum_{\mathrm{i}=1}^{\mathrm{k}} \beta_{i i} X_{i}^{2}+ \\
& \sum_{\mathrm{i}=1}^{\mathrm{k}-1} \sum_{\mathrm{j}=2}^{\mathrm{k}} \beta_{i j} X_{i} X_{j}+\varepsilon
\end{aligned}
$$

donde $Y$ es la respuesta del modelo, $k$ el número de factores, $\beta_{0}$ el coeficiente constante, $\beta_{i}$ el coeficiente lineal, $\beta_{i i}$ el coeficiente cuadrático, $\beta_{i j}$ el coeficiente de interacción, $X_{\mathrm{i}} \mathrm{y} X_{\mathrm{j}}$ las variables independientes (sustrato, inóculo y $\mathrm{pH})$, y $\varepsilon$ el error residual.

El análisis de varianza (ANOVA) y la metodología de superficie de respuesta se realizaron mediante el software Design Expert versión 7.0. Los valores de $p$ se utilizaron para indicar los términos significativos del modelo $(p<0.05)$ (Montgomery 2005).

Los datos obtenidos a partir del análisis de los AGV de los diferentes tratamientos fueron analizados mediante un ANOVA de una vía aplicando la prueba de Tukey con el software Minitab versión 17.0.

\section{RESULTADOS Y DISCUSIÓN}

\section{Desarrollo y análisis estadístico de la ecuación del modelo de regresión}

A partir del análisis de regresión múltiple sobre el conjunto de datos experimentales de la producción de biogás y rendimiento de metano que se muestra en el cuadro III, se establecieron dos modelos polinomiales de segundo orden (ecuaciones 2 y 3 ). A 
partir de ambos modelos se obtuvieron los valores óptimos de las variables de operación.

$$
\begin{aligned}
& Y_{\substack{\text { producción } \\
\text { Biogás }}}=312.83+27.67 X_{1}+20.42 X_{2}- \\
& 27.67 X_{3}+0.75 X_{1} X_{2}-3.62 X_{1} X_{3}-3.00 X_{2} X_{3}- \\
& 44.62 X_{1}^{2}-39.88 X_{2}^{2}-82.62 X_{3}^{2} \\
& Y_{\begin{array}{l}
\text { Rendimiento } \\
\text { Metano }
\end{array}}=433.46+23.13 X_{1}+1.12 X_{2}- \\
& 23.71 X_{3}+2.06 X_{1} X_{2}-6.81 X_{1} X_{3}+8.06 X_{2} X_{3}- \\
& 81.37 X_{1}^{2}-96.75 X_{2}^{2}-117.87 X_{3}^{2}
\end{aligned}
$$

El valor $\mathrm{F}$ obtenido para las ecuaciones 2 y 3 fue de 7.89 y 6.74 , respectivamente, lo cual indica que ambos modelos predicen adecuadamente los valores de producción de biogás y el rendimiento de metano. El valor de $p$ obtenido para las ecuaciones 2 y 3 , de 0.0004 y $0.0009(p<0.05)$, respectivamente, muestra que los resultados de ambos modelos son significativos para la producción de biogás y rendimiento de metano (Cuadro IV).

El ANOVA indicó que los coeficientes lineales de concentración inicial de inóculo y pH inicial, así como los tres coeficientes cuadráticos del modelo de producción de biogás fueron estadísticamente significativos (Cuadro IV). En este mismo sentido, el ANOVA también indicó que únicamente los tres coeficientes cuadráticos del modelo de rendimiento de metano fueron estadísticamente significativos (Cuadro IV).

\section{Análisis de superficie de respuesta}

En la figura 1 se muestran las gráficas tridimensionales de superficie de respuesta para producción de biogás y rendimiento de metano. La gráfica tridimensional de superficie de respuesta representa el modelo que describe el efecto y predice la respuesta para los diferentes valores de las variables independientes (Montgomery 2005, Wang y Wan 2009).

\section{Efecto de la concentración inicial de sustrato}

Los efectos interactivos en la producción de biogás y rendimiento de metano a partir del análisis comparativo de la variable de concentración inicial de sustrato respecto al $\mathrm{pH}$ y la concentración iniciales del inóculo se muestran en la figura 1a-d.

Las gráficas muestran que manteniendo constantes las condiciones óptimas de $\mathrm{pH}$ inicial de 7.1 y concentración inicial de inóculo de $22.4 \mathrm{~g} / \mathrm{L}$, la concentración inicial de sustrato de $0.5 \mathrm{~g} / \mathrm{L}$ no favoreció en gran medida la producción de biogás y el rendimiento de metano. No obstante, ambas variables

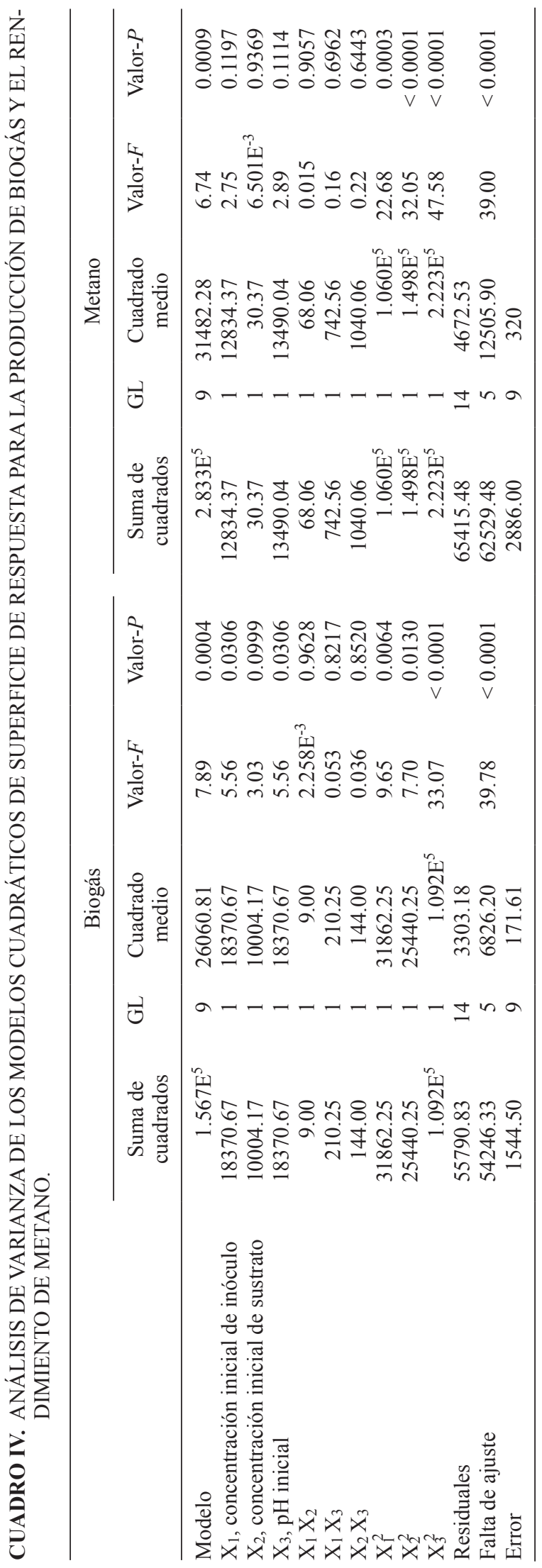



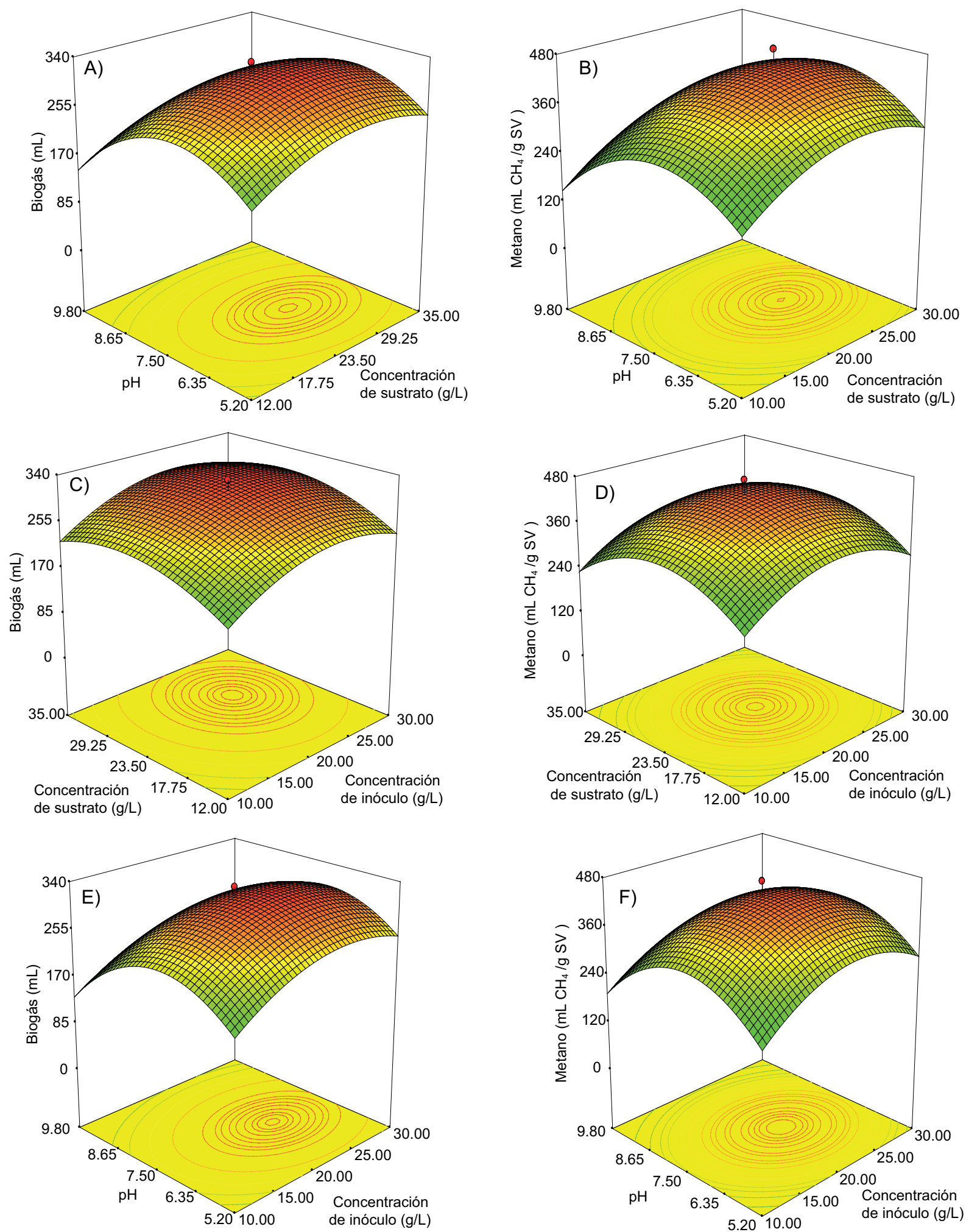

Fig. 1. Gráficos de superficie de respuesta tridimensional que representan el efecto de las condiciones iniciales de concentración de sustrato, concentración de inóculo y pH inicial, y su interacción con la producción de biogás y el rendimiento de metano. 
de respuesta aumentaron a partir del incremento de la concentración inicial de sustrato de 12 a $35 \mathrm{~g} / \mathrm{L}$, obteniéndose los valores más altos a $24.6 \mathrm{~g} / \mathrm{L}$. Sin embargo, el incremento en la concentración inicial de sustrato de 35 a $46.5 \mathrm{~g} / \mathrm{L}$ tuvo un efecto negativo en la producción de biogás y el rendimiento de metano, disminuyéndolos en un 38 y 80\%, respectivamente.

Sánchez et al. (2005) reportaron que una concentración limitada de sustrato en los procesos de digestión anaerobia tiende a causar una disminución en la actividad metabólica de los microorganismos. Zhang et al. (2014) observaron que con $0.5 \mathrm{~g} / \mathrm{L}$ de sustrato hay una limitación de carbono que tiende a afectar la tasa de crecimiento microbiano y la producción de biogás.

Los resultados del presente estudio mostraron que la producción de biogás y el rendimiento de metano disminuyeron a partir del incremento de la concentración de sustrato por encima del nivel óptimo. Este comportamiento puede deberse a que concentraciones de sustrato por encima del nivel óptimo inhiben el proceso de digestión anaerobia a causa de la reducción de $\mathrm{pH}$ por acumulación de AGV y amonio (Zhang et al. 2014, Polizzi et al. 2018).

\section{Efecto de la concentración inicial de inóculo}

En la figura 1c-f se muestran los efectos interactivos en la producción de biogás y rendimiento de metano, a partir del análisis comparativo de la variable de concentración inicial de inóculo respecto a la concentración inicial de sustrato y al $\mathrm{pH}$ inicial. Con base en las gráficas se muestra que manteniendo constante un $\mathrm{pH}$ inicial de 7.1 y una concentración inicial de sustrato de $24.6 \mathrm{~g} / \mathrm{L}$, el incremento en la concentración inicial de inóculo a partir de 10 a $30 \mathrm{~g} / \mathrm{L}$ tuvo un efecto positivo en la producción de biogás y el rendimiento de metano, obteniéndose los valores más altos a una concentración inicial de $22.4 \mathrm{~g} / \mathrm{L}$. Sin embargo, cuando esta concentración se incrementó a partir de 30 a $40 \mathrm{~g} / \mathrm{L}$, afectó la producción de biogás y el rendimiento de metano, los cuales disminuyeron en 40 y $63 \%$, respectivamente. Este comportamiento puede deberse a que la fase adaptación de los microorganismos disminuye a una elevada concentración de inóculo, lo cual induce una mayor producción de AGV y llega a provocar inhibición de la fase metanogénica (Zhou et al. 2011, Elbeshbishy et al. 2012, Bong et al. 2017).

En este estudio a concentraciones de inóculo por debajo del nivel óptimo se tuvo un efecto negativo en la producción de biogás y el rendimiento de metano, lo cual puede atribuirse a que las reacciones de biodegradación no ocurrirán si la cantidad de microorganismos no es suficiente, afectando la producción de biogás (Nazaitulshila et al. 2015).

\section{Efecto del pH inicial}

Los efectos interactivos en la producción de biogás y rendimiento de metano a partir del análisis comparativo del $\mathrm{pH}$ inicial respecto a la concentración inicial de sustrato y del inóculo, se muestran en la figura 1a, b, e y f. Con base en las gráficas se muestra que manteniendo constantes las condiciones óptimas de concentración inicial de sustrato a $24.6 \mathrm{~g} / \mathrm{L}$ y la concentración inicial de inóculo a $22.4 \mathrm{~g} / \mathrm{L}$, el pH inicial de 2.9 afectó la producción de biogás y el rendimiento de metano. Sin embargo, el incremento a partir del pH inicial de 5.2 a 7.5 tuvo un efecto positivo en dichos parámetros, obteniéndose los valores más altos a un $\mathrm{pH}$ inicial de 7.1. Contrariamente, el incremento a partir de un $\mathrm{pH}$ inicial de 7.1 a 9.8 condujo a una disminución de la producción de biogás de $35 \%$ y del rendimiento de metano de $32 \%$.

$\mathrm{Se}$ ha reportado que con un $\mathrm{pH}$ inferior de 6.0 disminuye la energía requerida para el intercambio de protones a través de la membrana celular, por tanto afecta el crecimiento celular y la producción de biogás (Russell y Dombrowski 1980, Chen et al. 2015). Deublein y Steinhauser (2008) reportaron que el pH óptimo para la producción de biogás está en el rango de 6.7 a 7.5. Valores inferiores a 6.7 pueden inducir considerable supresión del metabolismo en el proceso de digestión anaerobia, causando efectos negativos en la producción de biogás. Flores et al. (2020) reportaron que con un rango de $\mathrm{pH}$ de 7.0 a 8.0 en el proceso de digestión anaerobia del lactosuero se obtuvo la máxima producción de biogás y rendimiento de metano. El efecto negativo de valores de $\mathrm{pH}$ superiores a 8.0 en la producción de biogás y el rendimiento de metano puede obedecer a que favorecen la formación de subproductos metabólicos que tienen un efecto antagónico en la etapa de metanogénesis (Rajagopal et al. 2013)

En el cuadro I se comparan los valores máximos de producción de biogás y rendimiento de metano obtenidos a partir de condiciones óptimas con los reportados en otros estudios que usaron lactosuero como sustrato. Puede observarse que en este estudio se obtuvieron valores ligeramente más altos, lo cual indica que las condiciones óptimas favorecen el incremento en la producción de biogás mediante el proceso de digestión anaerobia del lactosuero.

\section{Verificación y validación del modelo}

Los valores iniciales de las condiciones óptimas de producción de biogás fueron los siguientes: 
concentración inicial de sustrato: $24.6 \mathrm{~g} / \mathrm{L}$; concentración inicial de inóculo: $22.4 \mathrm{~g} / \mathrm{L} ; \mathrm{pH}$ inicial: 7.1.

Para validar los resultados obtenidos se realizaron experimentos por duplicado usando las condiciones óptimas, en donde se obtuvo una producción de biogás y un rendimiento de metano de $331 \pm 1.13 \mathrm{~mL}$ y $469.75 \pm 3.25 \mathrm{~mL} \mathrm{CH}_{4} / \mathrm{g} \mathrm{SV}$, respectivamente. Estos resultados indican que ambos modelos polinomiales de segundo orden (ecuaciones 2 y 3 ) son adecuados para predecir la producción de biogás y el rendimiento de metano.

\section{AGV producidos durante la digestión anaerobia}

Los principales AGV analizados a las 168 y $336 \mathrm{~h}$ fueron acetato, propionato y butirato, representados de forma gráfica en la figura 2. De acuerdo con los resultados se muestra que a las $168 \mathrm{~h}$ el acetato se obtuvo en mayor concentración respecto a la concentración de propionato y butirato. Sin embargo, a las $336 \mathrm{~h}$ la concentración de propionato y butirato fue más alta con relación a la de acetato. Por lo tanto, los AGV producidos a las 168 y $336 \mathrm{~h}$ indican que la producción de biogás ocurre principalmente a partir de acetato y no de hidrógeno y $\mathrm{CO}_{2} \mathrm{o}$ formiato, sugiriendo que este metabolismo fue llevado a cabo por microorganismos metanogénicos acetoclásticos.

Se ha reportado en estudios de digestión anaeróbica con estiércol de vaca que el acetato es un intermediario metabólico necesario para la producción de biogás (Page et al. 2014). Demirel y Scherer (2008) y Jha et al. (2012) reportaron que la producción de metano en el biogás usando como inóculo estiércol
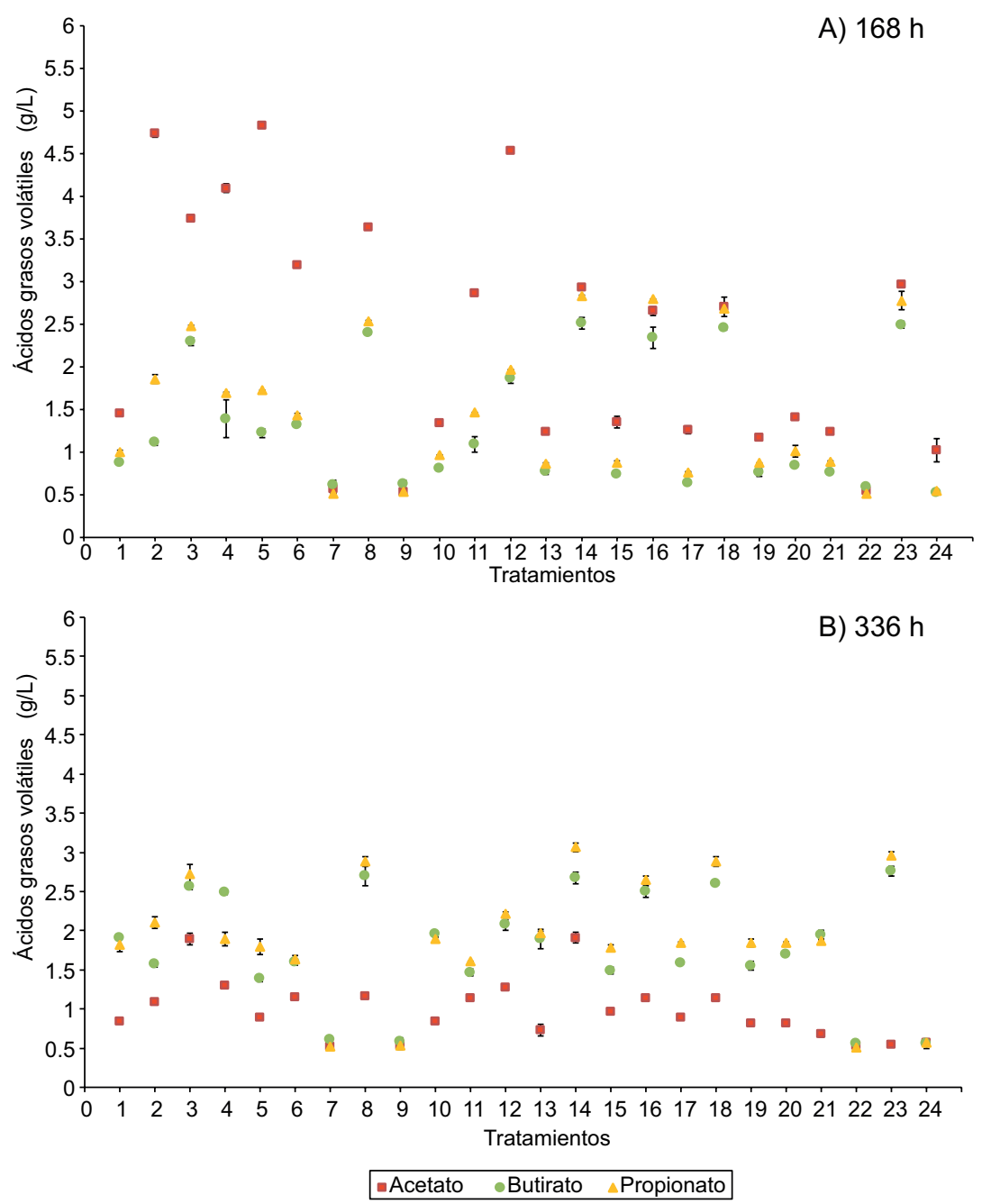

Fig. 2. Concentración de ácidos grasos volátiles producidos durante la digestión anaerobia del lactosuero para los diferentes tratamientos a las (a) $168 \mathrm{~h}$ y (b) $336 \mathrm{~h}$. 
de vaca se debió al consumo de acetato por microorganismos metanogénicos acetoclásticos en la etapa de metanogénesis. Los resultados de Achinas et al. (2018) señalan que el acetato fue uno de los metabolitos utilizados en mayor proporción por los microorganismos del estiércol de vaca en la producción de biogás, lo cual confirma los hallazgos del presente estudio.

\section{CONCLUSIONES}

En el presente estudio se aplicó un diseño experimental de optimización central compuesto al proceso de digestión anaerobia del lactosuero, para determinar los efectos y valores óptimos de concentración inicial de sustrato, concentración inicial de inóculo y $\mathrm{pH}$ inicial en la producción de biogás y rendimiento de metano. Los modelos heurísticos de producción de biogás y rendimiento de metano desarrollados en este estudio satisfacen todos los argumentos necesarios para su uso en la optimización. Este artículo proporciona detalladamente el análisis estadístico que se utilizó para determinar los niveles e interacciones óptimas entre las variables de proceso en la producción de biogás y el rendimiento de metano.

Los niveles óptimos de las variables de operación de este proceso de digestión anaerobia pueden ser una alternativa en el tratamiento de lactosuero en biodigestores a escala industrial para la producción de biogás, lo que favorecería al medio ambiente y reduciría los costos de producción.

\section{AGRADECIMIENTOS}

Agradecemos al Consejo Nacional de Ciencia y Tecnología (CONACYT) y a la Secretaría de Energía (SENER) por el financiamiento del proyecto número 261600. Los autores agradecen a grupo LALA por permitir el uso de sus instalaciones para el desarrollo del proyecto.

\section{REFERENCIAS}

Achinas S., Li Y., Achinas V. y Euverink G.J. (2018). Influence of sheep manure addition on biogas potential and methanogenic communities during cow dung digestion under mesophilic conditions. Sustain. Environ. Res. 28 (5), 240-246. https://doi.org/10.1016/j. serj.2018.03.003
Albalasmeh A.A., Berhe A.A. y Ghezzehei T.A. (2013). A new method for rapid determination of carbohydrate and total carbon concentrations using UV spectrophotometry. Carbohydr. Polym. 97 (2), 253-261. https:// doi.org/10.1016/j.carbpol.2013.04.072

APHA (2017). Standard methods for the examination of water and wastewater. 23a ed. American Public Health Association. Washington, EUA, 174 pp.

Antonelli J., Lindino C.A., de Azevedo J.C.R., de Souza S.N.M., Cremonez P.A. y Rossi E. (2016). Biogas production by the anaerobic digestion of whey. Rev. Ciênc. Agrár. 39 (3), 463-468. https://doi.org/10.19084/ RCA15087

Bezerra M.A., Santelli R.E., Oliveira E.P., Villar L.S. y Escaleira L.A. (2008). Response surface methodology (RSM) as a tool for optimization in analytical chemistry. Talanta 76 (5), 965-977. https://doi.org/10.1016/j. talanta.2008.05.019

Boboescu I.Z., Ilie M., Gherman V.D., Mirel I., Pap B., Negrea A., Kondorosi É., Bíró T. y Maróti G. (2014). Revealing the factors influencing a fermentative biohydrogen production process using industrial wastewater as fermentation substrate. Biotechnol. Biofuels 7 (139), 1-15. https://doi.org/10.1186/s13068-014-0139-1

Bong C.P., Tin Lee C., Shin Ho W., Hashim H., Klemeš J.J. y Siong Ho C. (2017). Mini-review on substrate and inoculum loadings for anaerobic co-digestion of food waste. Chem. Eng. Trans. 56, 493-498. https:// doi.org/10.3303/CET1756083

Burke P.J. y Yang H. (2016). The price and income elasticities of natural gas demand: international evidence. Energ. Econ. 59, 466-474. https://doi.org/10.1016/j. eneco.2016.08.025

Carrillo J., Celis L.B., Alatriste F. y Razo E. (2014). Decreasing methane production in hydrogenogenic UASB reactors fed with cheese whey. Biomass Bioenergy 63, 101-108. https://doi.org/10.1016/j.biombioe. 2014.01 .050

Carlini M., Castellucci S. y Moneti M. (2015). Biogas production from poultry manure and cheese whey wastewater under mesophilic conditions in batch reactor. Energy Procedia 82, 811-818. https://doi. org/10.1016/j.egypro.2015.11.817

Chatzipaschali A.A. y Stamatis A.G. (2012). Biotechnological utilization with a focus on anaerobic treatment of cheese whey: current status and prospects. Energies 5 (9), 3492-3525. https://doi.org/10.3390/en5093492

Chen S., Zhang J. y Wang X. (2015). Effects of alkalinity sources on the stability of anaerobic digestion from food waste. Waste Manage. Res. 33 (11), 1033-1040. https://doi.org/10.1177/0734242X15602965

Chun C.W., Mohd N.F. y Zainol N. (2015). Optimization of biogas production from poultry manure 
wastewater in $250 \mathrm{ml}$ flasks. J. Teknol. 75 (1), 275-285. https://doi.org/10.11113/jt.v75.3981

Comino E., Rosso M. y Riggio V. (2009). Development of a pilot scale anaerobic digester for biogas production from cow manure and whey mix. Bioresour. Technol. 100 (21), 5072-5078. https://doi.org/10.1016/j. biortech.2009.05.059

Comino E., Riggio, V.A. y Rosso M. (2012). Biogas production by anaerobic co-digestion of cattle slurry and cheese whey. Bioresour. Technol. 114 (2012), 46-53. https://doi.org/10.1016/j.biortech.2012.02.090

Council W.E. (2019). World energy scenarios 2019. Composing energy futures to 2050 [en línea]. https://www. worldenergy.org/assets/downloads/World-EnergyScenarios_Composing-energy-futures-to-2050_Fullreport1.pdf

Demirel B. y Scherer P. (2008). The roles of acetotrophic and hydrogenotrophic methanogens during anaerobic conversion of biomass to methane: a review. Rev. Environ. Sci. Bio. 7 (2), 173-190. https://doi.org/10.1007/ s11157-008-9131-1

Deublein D. y Steinhauser A. (2008). Biogas from waste and renewable resources. 2nd ed. Wiley-VCH, Alemania, 578 pp. https://doi.org/ 10.1002/9783527621705

Dudley B. (2019). BP statistical review of world energy [en linea]. https://www.bp.com/content/dam/bp/businesssites/en/global/corporate/pdfs/energy-economics/ statistical-review/bp-stats-review-2019-full-report.pdf

Elbeshbishy E., Nakhla G. y Hafez H. (2012). Biochemical methane potential (BMP) of food waste and primary sludge: influence of inoculum pre-incubation and inoculum source. Bioresour. Technol. 110, 18-25. https:// doi.org/10.1016/j.biortech.2012.01.025

Ergüder T., Tezel U., Güven E. y Demirer G. (2001). Anaerobic biotransformation and methane generation potential of cheese whey in batch and UASB reactors. Waste Manage. 21 (7), 643-650. https://doi. org/10.1016/S0956-053X(00)00114-8

Fernández C., Cuetos M.J., Martínez E.J. y Gómez X. (2015). Thermophilic anaerobic digestion of cheese whey: coupling $\mathrm{H}_{2}$ and $\mathrm{CH}_{4}$ production. Biomass Bioenergy 81, 55-62. https://doi.org/10.1016/j.biombioe.2015.05.024

Flores A.P., Hernández H., Cocotle Y. y Hernández E. (2020). Methanogenesis of raw cheese whey: $\mathrm{pH}$ and substrate-inoculum ratio evaluation at mesophyll temperature range. J. Chem. Technol. Biotechnol. 95 (7), 1946-1952. https://doi.org/10.1002/jctb.6391

Gómez T.F., Celis L.B., Razo E. y Alatriste F. (2012). Chemical and enzymatic sequential pretreatment of oat straw for methane production. Bioresour. Technol. 116, 372-378. https://doi.org/10.1016/j. biortech.2012.03.109
Hagos K., Zong J., Li D., Liu C. y Lu X. (2017). Anaerobic co-digestion process for biogas production: progress, challenges and perspectives. Renewable Sustainable Energy Reviews 76), 1485-1496. https:// doi.org/10.1016/j.rser.2016.11.184

IEA (2019). World energy outlook 2019. International Energy Agency [en línea]. https://www.iea.org/reports/ world-energy-outlook-2019

Jha A.K, Li J., Ban Q., Zhang L. y Zhao B. (2012). Dry anaerobic digestion of cow dung for methane production: effect of mixing. Pak. J. Biol. Sci. 15 (23), 1111-1118. https://doi.org/10.3923/pjbs.2012.1111.1118

Kim M.D., Song M., Jo M., Shin S.G., Khim J.H. y Hwang S. (2010). Growth condition and bacterial community for maximum hydrolysis of suspended organic materials in anaerobic digestion of food waste-recycling wastewater. Appl. Microbiol. Biotechnol. 85 (5), 16111618. https://doi.org/10.1007/s00253-009-2316-x

Kim M.J. y Kim S.H. (2017). Minimization of diauxic growth lag-phase for high-efficiency biogas production. J. Environ. Manage. 1 (187), 456-463. https://doi. org/10.1016/j.jenvman.2016.11.002

Lappa I.K., Papadaki A., Kachrimanidou V., Terpou A., Koulougliotis D., Eriotou E. y Kopsahelis N. (2019). Cheese whey processing: integrated biorefinery concepts and emerging food applications. Foods 8 (8), 1-37. https://doi.org/10.3390/foods8080347

Leung D.Y. y Wang J. (2016). An overview on biogas generation from anaerobic digestion of food waste. Int. J. Green Energy 13 (2), 119-131. https://doi.org/1 $0.1080 / 15435075.2014 .909355$

Liu G., Zhang R, El H.M., Dong R. y Liu X. (2012). Biogasification of green and food wastes using anaerobic-phased solids digester system. Appl. Biochem. Biotechnol. 168 (1), 78-90. https://doi.org/10.1007/s12010-011-9322-z

Mainardis M., Cabbai V., Zannier G., Visintini D. y Go D. (2017). Characterization and BMP tests of liquid substrates for high-rate anaerobic digestion. Chem. Biochem. Eng. Q. 31 (4), 509-518. https://doi. org/10.15255/CABEQ.2017.1083

Montgomery D.C. (2005). Diseño y análisis de experimentos. 2a ed. Limusa Wiley, México, 686 pp.

Najafpour G.D., Komeili M., Tajallipour M. y Asadi M. (2010). Bioconversion of cheese whey to methane in an upflow anaerobic packed bed bioreactor. Chem. Biochem. Eng. Q. 24 (1), 111-117. https://doi. org/10.15255/CABEQ.2014.266

Nazaitulshila R., Idris A., Harun R., y Wan Azlina W. (2015). The influence of inoculum to substrate ratio on the biochemical methane potential of fat, oil, and grease in batch anaerobic assays. Energ. Sources Part A. 37 (6), 590-597. https://doi.org/10.1080/1556703 6.2014 .907374 
Page L.H., Ni J.Q., Heber A.J., Mosier N.S., Liu X., Joo H.S., Ndegwa P.M. y Harrison J.H. (2014). Characteristics of volatile fatty acids in stored dairy manure before and after anaerobic digestion. Biosyst. Eng. 118, 16-28. https://doi.org/10.1016/j.biosystemseng.2013.11.004

Polizzi C., Alatriste F. y Munz G. (2018). The role of organic load and ammonia inhibition in anaerobic digestion of tannery fleshing. Water Resour. Ind. 19, 25-34. https://doi.org/10.1016/j.wri.2017.12.001.

Rajagopal R., Massé D.I. y Singh G. (2013). A critical review on inhibition of anaerobic digestion process by excess ammonia. Bioresour. Technol. 143, 632-641. https://doi.org/10.1016/j.biortech.2013.06.030

Ramos J.L., Vargas C.L., Mata J., y Camacho Á. (2019). Evaluation of poultry manure and goat cheese whey anaerobic co-digestion. Span. J. Agric. Res. 17 (2), 1-14. https://doi.org/10.5424/sjar/2019172-14577

Reungsang A, Pattra S. y Sittijunda S. (2012). Optimization of key factors affecting methane production from acidic effluent coming from the sugarcane juice hydrogen fermentation process. Energies 5 (12), 4746-4757. https://doi.org/10.3390/en5114746

Rico J.L., García P. y Fernández F. (1991). Anaerobic treatment of cheese-production wastewater using a UASB reactor. Bioresour. Technol. 37 (3), 271-276. https:// doi.org/10.1016/0960-8524(91)90194-O

Rico C., Muñoz N., Fernández J. y Rico J.L. (2015). Highload anaerobic co-digestion of cheese whey and liquid fraction of dairy manure in a one-stage UASB process: limits in co-substrates ratio and organic loading rate. Chem. Eng. J. 262, 794-802. https://doi.org/10.1016/j. cej.2014.10.050

Rosales L.M., Razo E., Ordóñez L.G., Alatriste F. y de León A. (2010). Hydrogen production by Escherichia coli $\triangle$ hycA $\Delta$ lacI using cheese whey as substrate. Int. J. Hydrogen Energy 35 (2), 491-499. https://doi. org/10.1016/j.ijhydene.2009.10.097

Russell J.B. y Dombrowski D.B. (1980). Effect of pH on the efficiency of growth by pure cultures of rumen bacteria in continuous culture. Appl. Environ. Microbiol. 39 (4), 604-610. https://doi.org/10.1128/ aem.39.3.604-610.1980
Saleh A.F., Kamarudin E., Yaacob A.B., Yussof A.W. y Abdullah M.A. (2012). Optimization of biomethane production by anaerobic digestion of palm oil mill effluent using response surface methodology. Asia-Pacific J. Chem. Eng. 7, 353-360. https://doi.org/10.1002/apj.550

Sánchez E., Borja R., Travieso L., Martin A. y Colmenarejo M. (2005). Effect of organic loading rate on the stability, operational parameters and performance of a secondary upflow anaerobic sludge bed reactor treating piggery waste. Bioresour. Technol. 96 (3), 335-344. https://doi.org/10.1016/j.biortech.2004.04.003

Sathish S. y Vivekanandan S. (2016). Parametric optimization for floating drum anaerobic bio-digester using response surface methodology and artificial neural network. Alexandria Eng. J. 55 (4), 3297-3307. https:// doi.org/10.1016/j.aej.2016.08.010

Scherer P. y Neumann L. (2013). "Methano-compost", a booster and restoring agent for thermophilic anaerobic digestion of energy crops. Biomass Bioenergy 56, 471478. https://doi.org/10.1016/j.biombioe.2013.05.021

Strydom J., Mostert J. y Britz T.J. (2001). Anaerobic treatment of a synthetic dairy effluent using a hybrid digester. Water S.A. 21 (2), 125-130.

Wang J. y Wan W. (2009). Experimental design methods for fermentative hydrogen production: a review. Int. J. Hydrogen Energy 34 (1), 235-244. https://doi. org/10.1016/j.ijhydene.2008.10.008

Wang J., Feng L., Tang X., Bentley Y. y Höök M. (2017). The implications of fossil fuel supply constraints on climate change projections: a supply-side analysis. Futures. 86, 58-72. https://doi.org/10.1016/j.futures.2016.04.007

Zhang W., Lang Q., Wu S., Li W., Bah H. y Dong R. (2014). Anaerobic digestion characteristics of pig manures depending on various growth stages and initial substrate concentrations in a scaled pig farm in Southern China. Bioresour. Technol. 156, 63-69. https://doi.org/10.1016/j.biortech.2014.01.013

Zhou Y., Zhang Z., Nakamoto T., Li Y., Yang Y., Utsumi M. y Sugiura N. (2011). Influence of substrate-toinoculum ratio on the batch anaerobic digestion of bean curd refuse-okara under mesophilic conditions. Biomass Bioenergy 35 (7), 3251-3256. https://doi. org/10.1016/j.biombioe.2011.04.002 